\title{
Liver foreign body resembling carcinoma
}

\author{
Giuseppe Re $\cdot$ Matteo Cescon • Benedetta Fabbrizio • \\ Rossella Romagnoli
}

Received: 7 March 2012 / Accepted: 5 May 2012/Published online: 25 May 2012

(C) SIMI 2012

\section{Background}

The occurrence of hepatic foreign body or bezoars is not a rare finding in patients with previous biliary enteric anastomosis or sphincterotomy, permitting the particle reflux from alimentary tract to bile ducts. On the other hand, the same condition is very rare when a choledochoenteric communication is not suggested by a history of abdominal surgery or trauma. An underlying communication may be present even if clinically unknown. This is the case of a silent abscess associated with chronic cholecystitis and forming a fistula into the liver with the passage of vegetal material. We describe such a rare case of a liver mass resembling tumor caused by the concretion of enteric material in a patient without a previous surgical treatment or endoscopic procedure.

G. Re $(\bowtie) \cdot$ R. Romagnoli

Department of Internal Medicine, Lugo Hospital,

Viale Dante 10, 48022 Lugo, RA, Italy

e-mail: g.re@ausl.ra.it

R. Romagnoli

e-mail: rossroma@libero.it

\section{Cescon}

Department of General Surgery and Organ Transplantation,

University of Bologna, Bologna, Italy

e-mail: matteo.cescon@unibo.it

B. Fabbrizio

Anatomy and Pathology Unit, University of Bologna,

Bologna, Italy

e-mail: benedetta.fabbrizio@aosp.bo.it

\section{Case presentation}

A 68-year-old man presented with abdominal discomfort and fever for 20 days. His medical history included noninsulin-dependent diabetes, hypertension and high LDL concentrations, which were currently treated with metformin, antiplatelet agents, antihypertensives and statins. He was a carrier of beta-thalassemia minor and he was a known case of long-standing chronic lithiasic cholecystitis. He had no history of abdominal surgery or trauma. At admission, body temperature was $39^{\circ} \mathrm{C}$. Laboratory investigations showed WBC count of $12,110 / \mathrm{mm}^{3}$ and C-reactive protein level of $239 \mathrm{mg} / \mathrm{L}$. Blood urea nitrogen and electrolytes were within normal ranges. Liver function tests were unremarkable (bilirubin: $0.49 \mathrm{mg} / \mathrm{dL}$; GGT: $68 \mathrm{U} / \mathrm{L}$; AST: $49 \mathrm{U} / \mathrm{L}$; ALT: $93 \mathrm{U} / \mathrm{L})$. Findings on physical examination included a mild hepatosplenomegaly, and a soft abdomen, albeit tender to deep palpation in the epigastrium, without evidence of rebound tenderness or peritoneal signs. Abdominal contrast ultrasonographic and computed tomographic scans showed an inhomogeneous irregular formation with ill-defined margins in the liver segments VIII and IV, suspicious for tumor with abscess transformation. Endoscopy of the upper gastrointestinal tract and colonoscopy, reaching the coecum, were performed, but no changes of the intestinal mucosa were found. Based on radiological findings, a liver resection was planned.

\section{Surgical treatment and outcome}

The patient was admitted to the Department of General Surgery and Organ Transplantation, University of Bologna. At laparotomy, neither ascites nor peritoneal tumor 
dissemination were detected. A large, whitish and hard mass, involving liver segments IV, V, and VIII, was discovered. At intraoperative ultrasound, this nodule was in contact with the middle hepatic vein, overtly invading the right anterior glissonian pedicle, and was adjacent to the right posterior portal branch, whose infiltration was highly suspected.

The gallbladder was atrophic and almost entirely included in the liver mass, strongly suggesting a neoplasm originating from the gallbladder and invading the liver. The second portion of the duodenum was apparently infiltrated by the tumor, and was partially resected. There was no invasion of the hepatic pedicle, and the stump of the cystic duct proved free from cancer invasion at intraoperative frozen section histology. A right hepatectomy extended to the anterior portion of segment IV was performed.

The early postoperative course was characterized by cholestasis, ascites, and alteration of liver function tests due to the small volume of the remnant liver. The patient was finally discharged on the 36th postoperative day with almost normal liver function and adequate hepatic regeneration detected by volumetric CT scan, being at present alive and in good general conditions.

\section{Pathological findings}

A histological assessment of the mass revealed vegetal material with some cellular debris. A fistula formation was assumed to have led to the development of the hepatic bezoar.

A right hepatectomy with micro-nodular external surface was examined. A $2 \mathrm{~cm}$ tract of small intestine was strictly fistulizated to peri-hilar hepatic structures. No gallbladder was found; the gallbladder hepatic's bed was occupied by a tubular structure, measuring $2.5 \mathrm{~cm}$ and showing amorphous and calcified appearance. On cut surface, the liver showed a $10 \mathrm{~cm}$ peri-hilar, wide area, yellowish-white coloured, with poorly defined margins and soft consistency, grossly suggestive of malignancy. The surrounding hepatic tissue showed altered lobular architecture, with multiple fine micro-nodules and dilation of major biliary ducts.

Surprisingly, the microscopic examination does not show a malignant process, but an extensive hepatic inflammatory reaction with wide areas of necrosis and abscesses formation, starting from the gallbladder hepatic's bed, with complete destruction of gallbladder wall and fistulisation into small intestine.

The gallbladder hepatic's bed was occupied by vegetal material, probably a large vegetal seed, surrounded by granulation tissue and a diffuse, chronic inflammatory, granulomatous reaction, with areas of necrosis, oedema and fibrosis (Fig. 1).

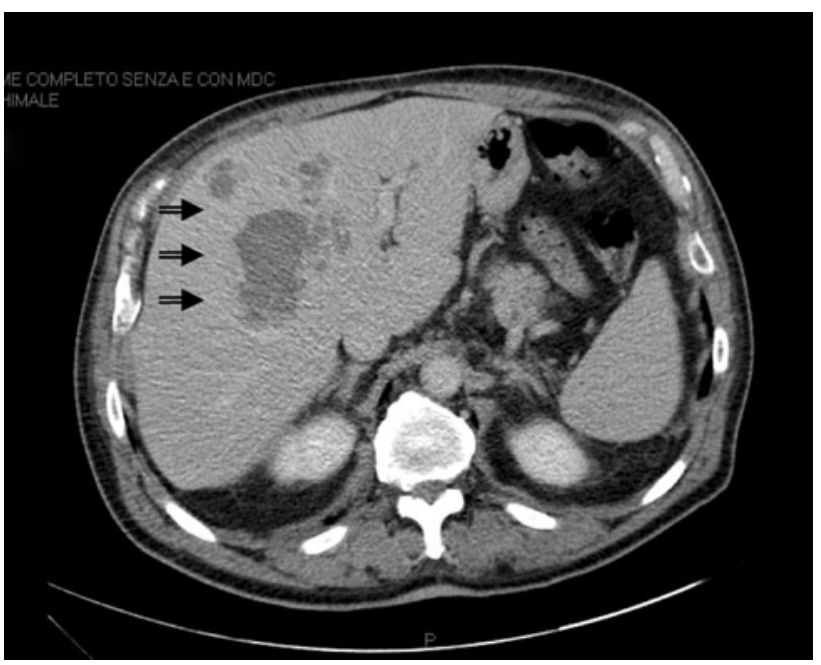

Fig. 1 CT scan images depicted a liver mass resembling a carcinoma (arrows), whereas the gallbladder remnant was not clearly visible, suggesting its complete encasement in the liver mass

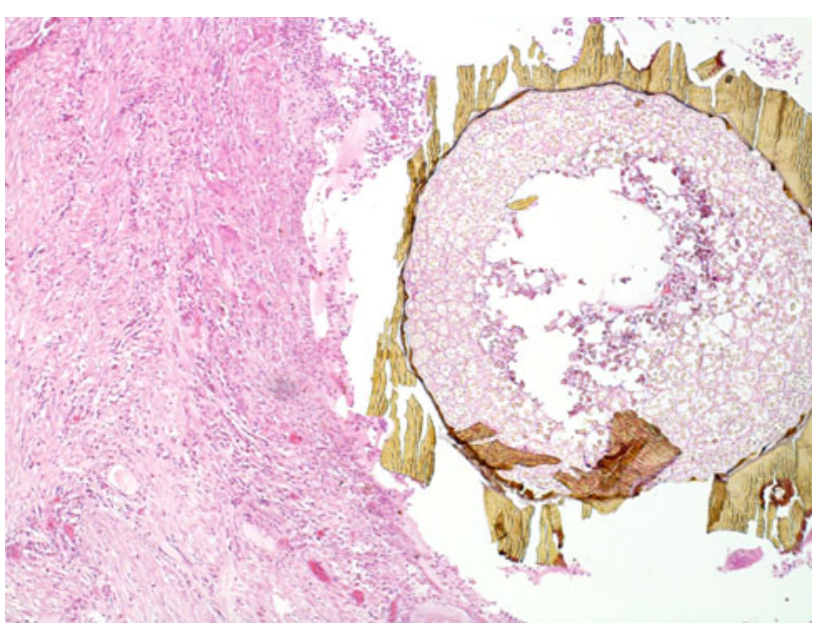

Fig. 2 Hepatic inflammatory reaction surrounding large vegetal seed

\section{Discussion}

Hepatic abscesses secondary to bezoar passage to liver from the intestinal tract are rare. The word "bezoar," paradoxically denoting counterpoison or antidote, derives from a greenish, hard concretion found in the fourth stomach of the Syrian goat, which was felt to prevent poisoning. Phytobezoars are simple concretions of poorly digested fruit and vegetable fibers in the alimentary tract. Most ingested bodies pass through the gastrointestinal tract without intestinal damage or complications (Fig. 2).

Migration of a phytobezoar to the liver may be a complication of any insult to enteric integrity. Perforation of the gastrointestinal tract may be secondary either to biliary stent migration [1] or to ingested foreign bodies, if they are 
fish or chicken bones. Cellulose or other previously unidentified material may be found in patients undergoing endoscopy for choledocholithiasis or with a previous surgical intervention [2-4]. Hepatic abscess due to bezoar translation, through a fistula formation, based on chronic cholecystitis, is a possible chain of events, but to our knowledge has not been prior reported.

In the present case, an entero-hepatic fistula was assumed to have led to the migration of the bezoar in the liver followed by the development of granulomatous inflammatory reaction surrounding the foreign body. Phytobezoar translation to the liver could be accounted for in the setting of a history of spontaneous fistula formation from a chronic cholecystitis. Without such a systematic account, preoperative diagnosis was difficult, and the surgical approach was accepted as the only effective, even in the absence of biopsy proven liver mass assessment. At laparotomy, the inflammatory mass could not be differentiated from a carcinoma, and in fact the conclusive diagnosis of bezoar was possible only at final pathology. On the other hand, surgery was totally necessary, since a recovery without a resection, even in the absence of tumor, was unpredictable. Surgical removal of the mass was the ultimate treatment.

To our knowledge, this is the first description of jejunal fistulisation secondary to chronic cholecystitis as the pathological basis for a bezoar passage to the liver. Whether the diabetes history also contributed in this case to the silent progression of the disease is not so clear but possible.

\section{Learning points}

- Migration of a phytobezoar from small bowel to liver is exceptional in patients without a history of abdominal surgery or endoscopic procedures. Before surgery, diagnosis is difficult as patients are generally unaware of the possible ingested body. The hepatic lesion appearance may be absolutely aspecific and the underlying foreign body may be underrecognized, as it was in our case.

- Ultrasound, endoscopy, CT and RM are necessary levels of investigation. Diagnostic signs defined on radiology imaging may be either non-specific or unremarkable or laconically suspicious for tumor with abscess transformation. The possibility that they may have no role when investigating liver lesion aetiology should be considered.

- Identification of the precise mechanism of liver insult often demands thorough consideration and surgical investigation.

\section{Conflict of interest None.}

\section{References}

1. Dawwas MF, Jah A, Griffiths WJH et al (2011) Image of the month—quiz case. Arch Surg 146(4):483

2. Santos SA, Alberto SC, Cruz E et al (2007) Hepatic abscess induced by foreign body: case report and literature review. World $\mathrm{J}$ Gastroenterol 13(9):1466-1470

3. Gundara S, Harrison R (2010) An unusual zoonosis: liver abscess secondary to asymptomatic colonic foreign body. HPB Surgery. ID 794271

4. Riani EB, Tancredi I, Sempoux C et al (2011) From interventional radiology to laparoscopic liver resection as complementary strategies in the treatment of hepatic abscess caused by ingested foreign bodies. Hepatogastroenterology 59(114): $558-560$ 